\title{
Resonant Phenomena in Antihydrogen-Hydrogen Scattering
}

\author{
A. Yu. Voronin ${ }^{1}$ and P. Froelich ${ }^{2}$ \\ 1 P.N. Lebedev Physical Institute, 53 Leninsky prospect, 117924 Moscow, Russia \\ ${ }^{2}$ Department of Quantum Chemistry, Uppsala University, \\ Box 518, SE-75120 Uppsala, Sweden.
}

\begin{abstract}
We present a treatment of cold hydrogen-antihydrogen collisions based on the asymptotic properties of atom-antiatom interactions. We derive general formulas for the elastic and inelastic cross sections and for the scattering lengths and analyze their sensitivity to the parameters characterizing the inelasticity of the collision process. Given the inelasticity, we obtain bounds for the complex scattering length. We investigate the influence of strong nuclear forces and the isotope effects in $\overline{\mathrm{H}} \mathrm{H}$ and $\overline{\mathrm{HD}}$ collisions and demonstrate enhancement of these effects due to the presence of the near-threshold narrow $\mathrm{HH}(\overline{\mathrm{HD}})$ states. The values of the elastic and inelastic cross-sections with simultaneous account of rearrangement and strong forces are presented. General expressions for the (complex) energies of the near-threshold $\mathrm{H} \overline{\mathrm{H}}$ states are obtained.
\end{abstract}




\section{INTRODUCTION}

A very important property of ultra-cold atom-antiatom collisions is the separation of scales, characteristic for inelastic transitions on one hand, and van der Waals interaction on the other hand. Indeed, the analysis of the dynamics of Protonium $(P n)$ and Positronium

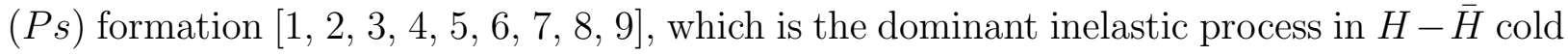
collisions, shows that such transitions happen mainly at internuclear separation distances less than $R_{s}$, which is on the order of the Hydrogen Bohr radius $\left(r_{B}\right)$. As shown by previous investigations [6] this distance is optimal for the overlap of the initial channel $(H-\bar{H})$ wave function and the final state $(P n-P s)$ wave-functions.

At separation distances larger than $R_{s}$ the $H-\bar{H}$ interaction is well approximated by the one-channel adiabatic potential $V_{a d}[9,10]$. Therefore the wave-function for the relative motion of the $H-\bar{H}$ could be obtained from the solution of the one-channel Schrödinger equation with the potential $V_{a d}(r)$, together with a proper boundary condition imposed at the internuclear distance $r \simeq R_{s}$. Importantly, such a boundary condition (which ideally should be derived from the solution of the four-body problem at internuclear distances $r<R_{s}$ ) turns out to be energy independent for sufficiently small energies of colliding $H$ and $\bar{H}$. Indeed, as follows from the kinematics of $P n$ and $P s$ formation [7] the energy of relative motion of $P n$ and $P s$ is $\varepsilon=E+M /\left(2 N^{2}\right)-m+m /\left(4 n^{2}\right)$, where $E$ is the $H-\bar{H}$ c.m. collision energy, $M$ is the reduced mass of $P n, m$ is the reduced mass of Hydrogen, $N$ is the principal quantum number of $P n, n$ is the principal quantum number of $P s$. As one can see the minimum of $\varepsilon$ is $\varepsilon_{m}=0.046$ a.u. $(E=0, N=24$ and $n=1)$. Therefore it is reasonable to expect that for $H_{-}^{-} H$ collision energies $E \ll \varepsilon_{m}$ the $P n$-Ps wave-function would weakly depend on the collisional energy $E$. In the following we will be interested in

cold collisions at energies $E \leq 10^{-5}$ a.u. where the S-wave contribution is dominant and the above condition is easily fulfilled. Thus the influence of rearrangement and annihilation on the wave-function in the $H \bar{H}$ channel can be described by the complex boundary condition for the logarithmic derivative of the wave-function at $R_{s}$, which is energy independent in the energy domain of interest. This boundary condition can be conveniently expressed in terms of the short-range complex phase-shift $\delta=\delta_{R}+i \delta_{I}$, which is connected to the above 
mentioned boundary condition via the relation

$$
\frac{\Phi^{\prime}\left(R_{s}\right)}{\Phi\left(R_{s}\right)}=p\left(R_{s}\right) \cot (\delta)
$$

where $p\left(R_{s}\right)=\sqrt{2 M V_{a d}\left(R_{s}\right)}$ is a classical local momentum given at the distance $R_{s}$.

In our study we will be interested in the near-threshold properties of $H-\bar{H}$ scattering. Such properties are critically dependent on the existence of the near-threshold singularities of the $S$-matrix, namely the weakly bound states or resonances. It is known that the density of the near-threshold levels is determined by the large distance behavior of adiabatic potential $V_{a d}(r)[11]$. In our case this potential at large distances turns into the homogeneous van der Waals potential $-C_{6} / r^{6}$. The strength of this potential $\left(C_{6}\right)$ determines the characteristic distance $R_{v d W}=\sqrt[4]{2 M C_{6}} \simeq 10.5$ a.u. which, as will be shown later, gives the order of magnitude of the scattering length for the $H \bar{H}$ system. At the same time the variation of the boundary condition (related to the short range phase-shift $\delta$, see eq. 1) will only weakly affect the density of the near-threshold levels; it results mainly in shifting of the whole spectrum of such states. It may happen that for certain value of $\delta$ there is a state (resonance) very close to the threshold. This results in a strong enhancement of the crosssections.

In this paper we will study the evolution of the scattering observables as a function of the short-range complex phase-shift $\delta$, thought of as a free variable. Though the "true" value of $\delta$ could be in principle obtained from the solution of the four-body problem, it is very instructive to get a general dependence of the scattering amplitude on $\delta$. Indeed, the existing model calculations of the low energy $H-\bar{H}$ scattering are all restricted by approximations in which the effect of certain decay channels are neglected. In terms of the here developed approach any account of additional channels, or generally any improvement of the optical potential, result in a change of the short range phase-shift $\delta$. We present the universal dependence of the scattering length on the complex short-range phase-shift $\delta$ in a closed form and show that such dependence manifests resonant behavior, connected to the existence of narrow near-threshold $H \bar{H}$ states. We analyze this behavior in terms of the near-threshold poles of the $S$-matrix. Such resonance phenomena can significantly enhance the influence of "small" physical effects (or their neglect in approximate treatments) and make inapplicable naive perturbation approach. In particular, we study the influence of strong forces in the non-relativistic $H \bar{H}$ Hamiltonian, including the shift and splitting of the 
quasi-bound near-threshold $H \bar{H}$ states, as well as the isotope effect in $D-\bar{H}$ scattering.

\section{LOW ENERGY S-STATE $H-\bar{H}$ SCATTERING}

We start with the derivation of the analytical expression for the scattering length for $H-$ $\bar{H}$ collisions. This derivation is based on the matching of the WKB form of the wave-function and the analytical zero-energy solution of the Schrödinger equation with the homogeneous $\left(-C_{6} / r^{6}\right)$ potential in the asymptotic region. Such an approach was successfully used for the study of cold atomic collisions [2, 12, 13]. Our treatment will be extended to the atomantiatom case, where account of inelastic transitions is important.

Further analysis is based on the fact, that the WKB approximation is applicable for the description of the wave-function in between the short-range distance and the asymptotic domain $R_{s}<r \ll R_{v d W}$ :

$$
\Phi(r) \sim \frac{1}{\sqrt{p(r)}} \sin \left(\delta+\int_{R_{s}}^{r} p\left(r^{\prime}\right) d r^{\prime}\right)
$$

where $p(r)=\sqrt{2 M V_{a d}(r)}$ is the classical local momentum in the adiabatic potential $V_{a d}(r)$, while $\delta=\delta_{R}+i \delta_{I}$ is the complex short range phase-shift, whose value should be fixed by matching with the solution of the four-body problem at $r=R_{s}$.

It is important that the distance $\left(R_{h}\right)$ above which the adiabatic potential $V_{a d}(r)$ is well approximated by the homogeneous $-C_{6} / r^{6}$ potential is within the distance of the validity of WKB approximation, i.e. $R_{h}<R_{v d W}$. The rough estimation of $R_{h}$ can be obtained from the condition that for the distances above $R_{h}$ the term $C_{6} / r^{6}$ should dominate in the multipole expansion of $V_{a d}(r)$. This gives $R_{h}=\sqrt{C_{8} / C_{6}} \sim 5$ a.u.. Thus there exists a matching region $R_{h}<r \ll R_{v d W}$, where both the WKB approximation and the zero-energy solution of the Schrödinger equation with homogeneous $\left(-C_{6} / r^{6}\right)$ potential are valid approximations of the $H \bar{H}$ wave-function.

In the vicinity of $R_{h}$, where the adiabatic potential is well reproduced by a homogeneous potential, the classical momentum is $p(r)=\sqrt{2 M C_{6} / r^{6}}$ and one can get the explicit $r$ dependence of the wave-function introduced in eq. (2):

$$
\Phi(r) \sim \frac{r^{3 / 2}}{\sqrt[4]{2 M C_{6}}} \sin \left(\delta+\Omega-\frac{\sqrt{2 M C_{6}}}{2 r^{2}}\right)
$$


where we have introduced the semiclassical phase $\Omega$ defined as

$$
\Omega=\int_{R_{s}}^{\infty} p(r) d r
$$

The value of $\Omega$ calculated using the adiabatic potential $V_{a d}$ of the $H-\bar{H}$ system from the ref. [14] and $R_{s}=1$ a.u. turns out to be $\Omega=19.383$.

The wave function given in eq. (3) can be matched in the vicinity of $R_{h}$ with the exactly known zero-energy wave function $\Phi_{0}(r)$ in a homogeneous potential $1 / r^{6}$ [15], which is a linear combination of the form:

$$
\Phi_{0}(r) \sim \sqrt{r}\left(J_{1 / 4}\left(\frac{\sqrt{2 M C_{6}}}{2 r^{2}}\right)-C Y_{1 / 4}\left(\frac{\sqrt{2 M C_{6}}}{2 r^{2}}\right)\right) .
$$

The coefficient $C$ has to be determined from the matching procedure at $R \simeq R_{h}$ which results in the following wave-function at distances $r>R_{h}$ :

$$
\Phi\left(r>R_{h}\right) \sim \sqrt{r}\left(J_{1 / 4}\left(\frac{\sqrt{2 M C_{6}}}{2 r^{2}}\right)-\tan \left(\frac{\pi}{8}+\Omega+\delta\right) Y_{1 / 4}\left(\frac{\sqrt{2 M C_{6}}}{2 r^{2}}\right)\right) .
$$

Using the Taylor expansion of the Bessel functions for small argument and taking into account that the scattering length appears in the asymptotic form of the wave function through

$$
\Phi(r \rightarrow \infty) \sim 1-r / a
$$

we obtain for the scattering length

$$
a=a_{0}\left(1+\cot \left(\frac{\pi}{8}+\Omega+\delta\right)\right)
$$

where $a_{0}$ is

$$
a_{0}=R_{v d W} \frac{\Gamma(3 / 4)}{2 \sqrt{2} \Gamma(5 / 4)} \simeq 4.99 \text { a.u.. }
$$

As one can see $R_{v d W}$ gives the characteristic size scale for the $H-\bar{H}$ scattering length. The argument of cotangent in (17) has a simple meaning. Indeed, $\Omega$ is the semiclassical phase accumulated in the region $r>R_{s}, \delta$ is the short range phase-shift accumulated at $r<R_{s}$ and the term $(\pi / 8)$ is the quantum correction to the semiclassical phase from the asymptotic van der Waals tail $\left(-C_{6} / r^{6}\right)$.

The accuracy of the above expression is limited by the accuracy of the WKB approximation in the range $R_{s}<r<R_{v d W}$ and on possible discrepancies between the adiabatic 
potential $V_{a d}$ and the van der Waals term $-C_{6} / r^{6}$ at the upper limit of that range. The comparison of (7) with the numerical calculation of the Schrödinger equation with the adiabatic potential $V_{a d}(r)$ and boundary condition (11) shows that formula (77) has the accuracy of $10 \%$ in the wide range of $\delta$.

One can extend the above analysis to the calculation of the effective range. According to [16, 17] the effective range is:

$$
r_{e}=\frac{a_{0}}{3}\left[\frac{\Gamma(1 / 4)}{\Gamma(3 / 4)}\right]^{2}\left(1-2 \frac{a_{0}}{a}+2 \frac{a_{0}^{2}}{a^{2}}\right) .
$$

Upon substitution of $a$ from eq. (7) one gets

$$
r_{e}=\frac{a_{0}}{3}\left[\frac{\Gamma(1 / 4)}{\Gamma(3 / 4)}\right]^{2} \frac{(\cot (\pi / 8+\Omega+\delta))^{2}+1}{(\cot (\pi / 8+\Omega+\delta)+1)^{2}} .
$$

For sufficiently low energy, i.e. when the momentum of the incident atom satisfies $k|a| \ll$ 1 the elastic $\left(\sigma_{e l}\right)$ and inelastic $\left(\sigma_{i n}\right)$ cross-sections are determined by the scattering length:

$$
\begin{aligned}
\sigma_{e l} & =4 \pi|a|^{2}=4 \pi a_{0}^{2}\left[\left(1+\operatorname{Re} \cot \left(\frac{\pi}{8}+\Omega+\delta\right)\right)^{2}+\left(\operatorname{Im} \cot \left(\frac{\pi}{8}+\Omega+\delta\right)\right)^{2}\right], \\
\sigma_{i n} & =4 \pi|\operatorname{Im} a| / k=4 \pi \frac{a_{0}}{k} \operatorname{Im} \cot \left(\frac{\pi}{8}+\Omega+\delta\right) .
\end{aligned}
$$

These values can be corrected to account for the second order terms in momentum $k$ :

$$
\begin{aligned}
\sigma_{e l} & =\frac{4 \pi|a|^{2}}{1-2 k \operatorname{Im} a+k^{2}\left(|a|^{2}-\operatorname{Re}\left(r_{e} a\right)\right)}, \\
\sigma_{i n} & =\frac{4 \pi}{k} \frac{|\operatorname{Im} a|}{1-2 k \operatorname{Im} a+k^{2}\left(|a|^{2}-\operatorname{Re}\left(r_{e} a\right)\right)} .
\end{aligned}
$$

Since the formation of Protonium and Positronium in the rearrangement collisions ends in complete annihilation, the total inelastic cross-section can be identified with the cross-section for annihilation.

We will now turn to the study of the general dependence of the scattering lengths and cross-sections (elastic and inelastic) on the variation of the real $\left(\delta_{R}\right)$ and imaginary $\left(\delta_{I}\right)$ parts of the short range phase-shift $\delta$.

Let us treat two important limiting cases. The first, which we refer to as the "weak absorption limit", is characterized by small imaginary phase-shift $\delta_{I} \ll 1$ which means that the probability of transitions to the inelastic channels is small. In that case we get for the scattering length $a$ from eq. (7)

$$
a=a_{0}\left(1+\cot \left(\frac{\pi}{8}+\Omega+\delta_{R}\right)\right)-i a_{0} \delta_{I} / \sin ^{2}\left(\frac{\pi}{8}+\Omega+\delta_{R}\right),
$$




$$
\begin{aligned}
& \sigma_{e l}=4 \pi|a|^{2}=2 \pi a_{0}^{2} \frac{\sin ^{2}\left(\frac{3 \pi}{8}+\Omega+\delta_{R}\right)}{\sin ^{2}\left(\frac{\pi}{8}+\Omega+\delta_{R}\right)} \\
& \sigma_{i n}=4 \pi|\operatorname{Im} a| / k=4 \pi \frac{a_{0}}{k} \frac{\delta_{I}}{\sin ^{2}\left(\frac{\pi}{8}+\Omega+\delta_{R}\right)}
\end{aligned}
$$

The scattering cross sections (eqs. 15-16) show oscillating character as a function of $\delta_{R}$. As will become apparent later such oscillations originate from the existence of a spectrum of long-lived near-threshold states of the $H \bar{H}$ system.

For the application to collisional cooling we are interested in the competition between the rate of elastic scattering and the rate of annihilation. The ratio $\sigma_{e l} / \sigma_{i n}$ reaches its maximum

$$
\left(\frac{\sigma_{e l}}{\sigma_{i n}}\right)_{\max }=\frac{a_{0} k}{2 \delta_{I}}
$$

for $\frac{\pi}{8}+\Omega+\delta_{R}=\pi / 4+\pi v, v=0,1, \ldots$ and its minimum

$$
\left(\frac{\sigma_{e l}}{\sigma_{i n}}\right)_{\min }=2 k a_{0} \delta_{I}
$$

for $\frac{\pi}{8}+\Omega+\delta_{R}=3 \pi / 4+\pi v, v=0,1, \ldots$.

Let us now turn to the opposite limit of strong absorption, characterized by $\delta_{I} \gg 1$. In this case we get

$$
\begin{aligned}
a & =a_{0}(1-i), \\
r_{e} & =0, \\
\sigma_{e l} & =8 \pi a_{0}^{2}, \\
\sigma_{i n} & =4 \pi \frac{a_{0}}{k} .
\end{aligned}
$$

The above cross-sections (eqs. 21-22) are determined by the van der Waals tail of the adiabatic potential only. Remarkably, they include no information about the short- and middle-range parts of the atom-antiatom interaction. All particles that penetrate to these short distances are lost, so the only information available to the observer is due to the so called quantum reflection [18, 19] from the asymptotic tail of the potential, which is determined by $C_{6}$ alone. A remarkable feature of the strong absorption limit is that the effective range (9) is exactly zero.

In Fig. 1 we plot the elastic cross-section as a function of real part of short-range phaseshift $\delta_{R}$ for two values of the "inelasticity parameter" $\delta_{I}=0.3$ and $\delta_{I}=0.7$. Pronounced 


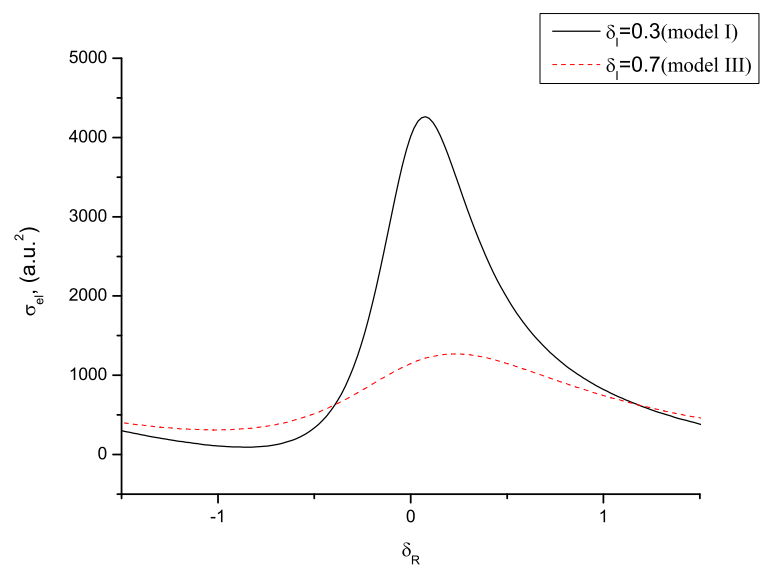

FIG. 1: Elastic cross-section for $H^{-} H$ collisions as a function of phase $\delta_{R}$, calculated at $E \rightarrow 0$ according to $\sigma_{e l}=4 \pi|a|^{2}$ with $a$ given by eq. (7).

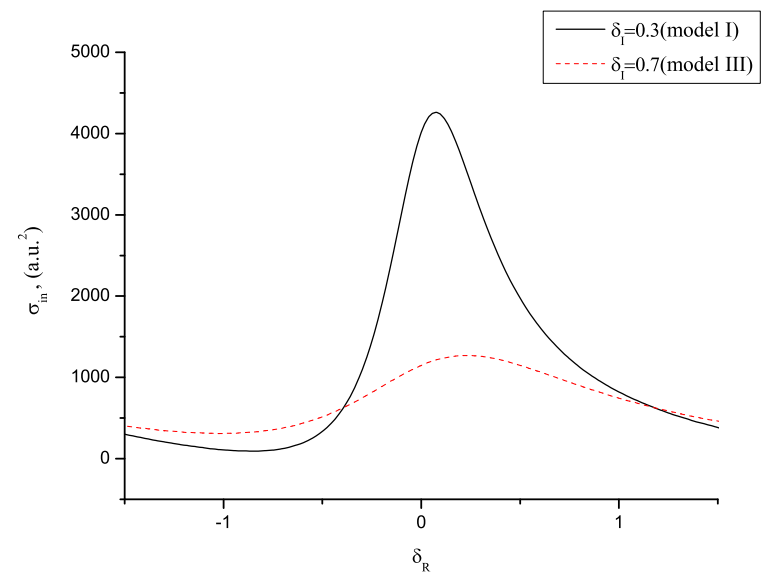

FIG. 2: Annihilation cross-section for $H-\bar{H}$ collisions as a function of phase $\delta_{R}$, calculated at the energy $\mathrm{E}=10^{-6}$ a.u. according to $\sigma_{i n}=4 \pi \operatorname{Im}(a) / k$ with $a$ given by eq. (17).

oscillations can be seen in the "weak absorption" case, which become much more smooth with increasing inelasticity parameter (in the limit $\delta_{I} \gg 1$ the cross-sections are constant). The same tendency is apparent in the inelastic cross-section (Fig. 2) and in the ratio of the elastic to the annihilation cross-section (Fig. 3). 


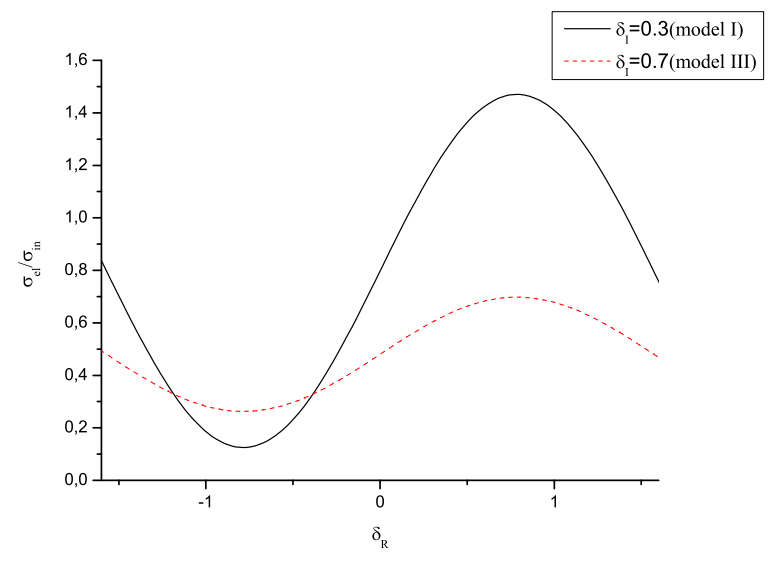

FIG. 3: Ratio of the elastic to the annihilation cross-section as a function of phase $\delta_{R}$, calculated at the energy $\mathrm{E}=10^{-6}$ a.u. with $a$ given by eq. (7).

\section{PHYSICAL IMPLICATIONS}

In this section we discuss the physical implications of the sensitive dependence of the scattering length on the short-range phase shift. We study the evolution of the scattering length as function of the phase-shift $\delta_{R}$. This can be considered as an universal tool for studying the contribution of various physical effects to scattering observables.

\section{Limits on the accuracy of the model $H_{1 S}-\bar{H}_{1 S}$ calculations}

Since the complete $a b$ initio treatment of the hydrogen-antihydrogen scattering is a difficult problem it is important to be able to estimate the accuracy of the approximate calculations. Several model calculations have been performed to get elastic and annihilation crosssections in the case of $H_{1 S}-\bar{H}_{1 S}$ scattering [3, 4, 5, 7, 8, 20, 21, 22, 23, 24, 25, 26, 27, 28]. In these models mostly the same adiabatic potential $V_{a d}$ of $H_{1 S}-\bar{H}_{1 S}$ was used, applicable in the distance range $r>R_{s} \simeq 1 r_{B}$ [14]. At the same time very different approximations were made regarding the rearrangement and strong forces. They include calculations of the elastic cross-section neglecting both rearrangement and strong force [7], the account of rearrangement in the "distorted-wave" approximation [6, 21, 29], calculation of the annihilation cross-section due to strong force only [21, 23], non-perturbative calculations based on the approximative optical potential [5, 8] and other. 
In terms of the formalism developed in the previous section the above mentioned models differ by the short-range phase $\delta=\delta_{R}+\delta_{I}$, i.e. the phase accumulated up to $R \simeq R_{s}$. This phase incorporates the effects of inelasticity due to strong forces and rearrangement. In the optical model calculations by Voronin and Carbonel [5] (further referred to as model I) it was found that $a=5.2-i 1.8$ a.u. (without account of strong force). From this value of the scattering length the corresponding value of the short-range phase-shift can be uniquely deduced (in the sense that the solution of the Schrödinger equation with given adiabatic potential $V_{a d}$ and with boundary condition (II) results in unique correspondence between $\delta$ and $a$, as can be seen from eq. (77)). The value of the short range phase-shift for model I turns out to be $\delta=0.696+i 0.305$. The value presented in [22] by Armour, Liu and Vigier (further referred to as model II) is $a=8.2-i 2.8$ a.u. and the short-range phase-shift deduced from this value of the scattering length is $\delta=0.136+i 0.41$. The optical model calculation [8] by Zygelman, Saenz, Froelich and Jonsell (further referred to as model III) gives $a=5.6-i 3.7$ a.u. (without strong force). The corresponding short-range phase-shift turns out to be $\delta=0.638+i 0.715$. Finally, the calculation of elastic scattering neglecting both rearrangement and strong force effects (further referred to as model IV, [29]) results in the scattering length $a=7.69$ a.u.. The corresponding real phase-shift, deduced from this value of the scattering length is $\delta=0.287$. All these values are tabulated in Table प. Inclusion of the inelasticity is thus equivalent to introduction of the short-phase corrections to model IV. Whereas it was possible to separately calculate the complex phase due to direct annihilation [21, 30], calculation of such phase correction due to the rearrangement is still incomplete since it is based only on the imaginary component of the optical potential [8]. Hence the inaccuracy of the real phase $\delta_{R}$ remains an important source of the inaccuracy of the scattering length in $H-\bar{H}$ scattering.

From the eqs. (15/16) it is clear that the smaller is the inelasticity parameter $\delta_{I}$, the more sensitive are the model results to the uncertainty in the real part of the $H-\bar{H}$ interaction at distances $r<R_{s}$. We notice that for a fixed value of inelasticity parameter $\delta_{I}$ the relation between imaginary and real part of the scattering length can not be arbitrary but is determined by eq. (7). To illustrate this statement we plot the possible values of the scattering length $a$ in the complex plane of $a$ as a function of $\delta_{R}$ (which changes from $-\pi / 2$ to $\pi / 2)$ for fixed value of $\delta_{I}$. The possible values of $a$ for each fixed value of $\delta_{I}$ form closed curves. The three curves of the scattering length correspond to three different inelasticity 


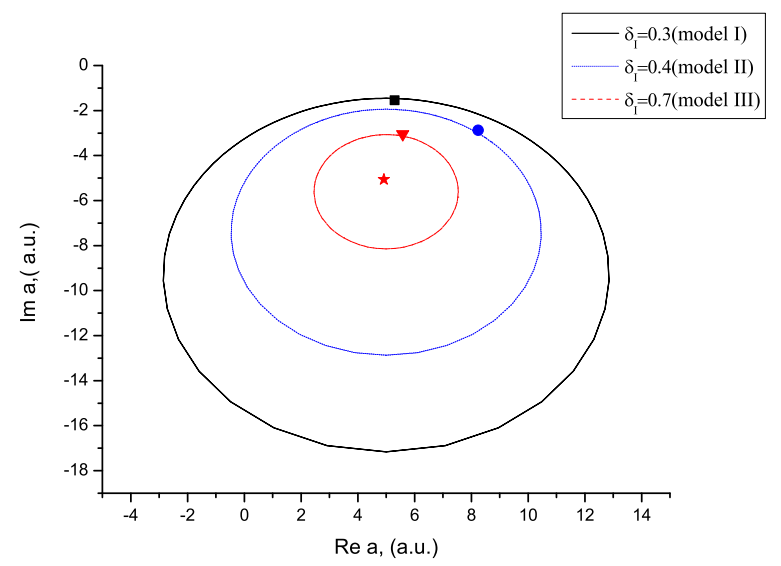

FIG. 4: Complex scattering length $a$ as function of the short-range phase-shift $\delta_{R}$, for the fixed value of $\delta_{I}$. The square indicates the complex value of the scattering length obtained in model I, circle - in model II, triangle - in model III. The value of $a \simeq 5-i 5$ a.u. marked by a star corresponds to the limiting case of strong absorption $\delta_{I} \gg 1$.

parameters $\delta_{I}$ deduced from models I-III. One can see that the smaller is $\delta_{I}$ the larger is the possible variation of $a$ with $\delta_{R}$. In the limit of zero absorption $\left(\delta_{I} \rightarrow 0\right)$ the variation becomes infinite and the closed curve degenerates into the whole real axis of the complex plane (model IV), while in the opposite limit of strong absorbtion the curve shrinks into a point $a=a_{0}(1-i) \simeq 5-i 5$ a.u.. Interestingly, the mean value of the scattering length $\bar{a}$ (averaged over $\delta_{R}$ ) is the same for all curves, i.e. it turns out to be independent on the inelasticity parameter $\delta_{I}$ and is equal to $\bar{a}=a_{0}(1-i)$.

The corresponding variations of the cross-sections are shown on Fig. 1 and Fig. 2 for models I and III (the corresponding inelasticity parameters are $\delta_{I}=0.3$ and $\delta_{I}=0.7$, respectively). One can see that the ratio of maximum to minimum possible values of elastic cross-section turns out to be $\sigma_{e l}^{\max } / \sigma_{e l}^{\min } \simeq 46$ for $\delta_{I}=0.3$. The same value for $\delta_{I}=0.7$ is much less and turns out to be $\sigma_{e l}^{\max } / \sigma_{e l}^{\min } \simeq 4$. In view of this result it is clear that the knowledge of the absorptive phase-shift $\delta_{I}$ is very essential and it is sufficient for establishing the limits of accuracy in calculations of both inelastic and elastic cross-sections.

We can perform further analysis and estimate how the uncertainty in the $H-\bar{H}$ interaction at distances $r<R_{s}$ would be reflected in the uncertainty of the scattering observables. Such an estimation can not be model independent. We will use the following simple assump- 
tions. One can expect [21, 22, 28] that the leading contribution to the mentioned interaction at distances $R<R_{s}$ is given by the local interaction of the form (expressed in a.u.):

$$
V_{l o c}(r)=-1 / r+0.75 \text {. }
$$

This potential continuously matches with $V_{a d}(r)$ at $r \approx R_{s}$ a.u. Apart from such local potential there is an additional interaction in the $H-\bar{H}$ channel, which results from coupling to the decay channels. We will model the effects of coupling to the $P n$ and $P s$ channels, responsible for rearrangement, by the local complex potential of the form:

$$
V_{o p t}=\frac{(v-i w)}{r_{B}} \exp (-2 r)
$$

The above simple form of "optical" potential is only used to mimic the effect of coupling to the decay channels and can not be treated as true form of effective short-range $H-\bar{H}$ interaction - but it captures its localization. The $a b$ initio calculations of complex nonlocal optical potential (so far restricted by approximations) can be found in [5, 8, 26]. These optical potentials are constructed by projecting the Hamiltonian on the subspace of open $P n$ and $P s$ channels and therefore their characteristic range is equal to the "size" of the $P n$ states with energetically highest possible principal quantum numbers $(N \leq 24)$. Because of that such ab initio optical potentials are localized below $R_{s}$ and this property is reflected in the $r$ dependence of the simple model potential $V_{\text {opt }}(r)$.

To calculate the short-range phase-shift we will use the semiclassical approximation for the phase:

$$
\delta \approx \int_{0}^{R_{s}} \sqrt{-2 M\left[V_{l o c}(r)+V_{o p t}(r)\right]} d r-\pi / 4 .
$$

The term $\pi / 4$ is the quantum correction to account for the correct behavior of the coulomb wave-function at small distances $r<1 / M$, where semiclassical approximation is no longer valid. Expecting $\left|V_{\text {opt }}(r)\right| \ll\left|V_{l o c}(r)\right|$ we get from (25) for $\delta$ :

$$
\begin{aligned}
\delta & =\delta^{0}+\delta^{o p t} \\
\delta^{0} & =\int_{0}^{R_{s}} \sqrt{-2 M V_{l o c}(r)} d r, \\
\delta^{o p t} & =-\sqrt{M} \int_{0}^{R_{s}} \frac{V_{o p t}(r)}{\sqrt{-2 V_{l o c}(r)}} d r .
\end{aligned}
$$

In the above expression $\delta^{0}$ is a phase-shift produced by the local potential $V_{l o c}$ alone, while $\delta^{o p t}$ is the variation of that phase-shift due to the presence of the optical potential. Using 
the adopted expressions for $V_{l o c}$ and $V_{\text {opt }}$ we come to the following numbers: $\delta^{0}=72.45$, $\delta^{o p t}=-(v-i w) 6.38$.

The corresponding variation of the scattering length can be obtained from eq. (14):

$$
\delta a=-a_{0} \frac{\delta^{o p t}}{\sin ^{2}\left(\pi / 8+\Omega+\delta^{0}+\delta^{o p t}\right)} .
$$

From the above numbers we can conclude, that the value of inelasticity parameter $\delta_{I}$ consistent with models I-III corresponds to the value of the imaginary part of the optical potential $w$ which lies in-between 0.05 and 0.1 a.u.

On Fig. 5 and Fig. 6] we plot the elastic and inelastic cross-sections as function of the real part of the model optical potential $V_{\text {opt }}$. Even a small and confined real part produces large variations of the cross sections. For the optical potential given by eq. (24), the mean variation of the ratio $\frac{R e V_{o p t}}{V_{l o c}}$ within the interval $\left[0, R_{s}\right]$ is on the order of $1 \%$, yet it produces more than $100 \%$ change of elastic and inelastic scattering-cross sections. This result makes clear the relative importance of the real part of the effective $H-\bar{H}$ shortrange interaction and puts obvious restrictions on the accuracy of calculations. It means in particular that the non-perturbative account of additional decay channels could result in significant changes of the elastic and inelastic cross-sections. We will show in the following that such an "instability" of model calculations (for relatively small values of inelasticity parameter $\delta_{I}$ ) is due to the existence of the spectrum of narrow near-threshold states in the $H-\bar{H}$ channel.

\section{Sensitivity to the nuclear interaction}

An important consequence of the weak absorption in $H_{1 S}-\bar{H}_{1 S}$ interaction is the possibility of observing the nuclear effects on the molecular scale. The effect of the nuclear forces was reported in ref. [5, 21, 23, 27] for the case of hydrogen-antihydrogen scattering and in ref. [30] for antihydrogen-helium scattering. In calculations [21, 22] no rearrangement effects were taken into account.

The strong force effects can be studied by inclusion of the complex short-range nuclear potential in the nonrelativistic Hamiltonian describing the $H \bar{H}$ system. This potential accounts for the nuclear interaction of proton and antiproton on the scale of few fm $\left(1.88 \cdot 10^{-5}\right.$ a.u.) and is chosen to reproduce the nuclear $p \bar{p}$ (spin dependent) scattering length. Incorpo- 


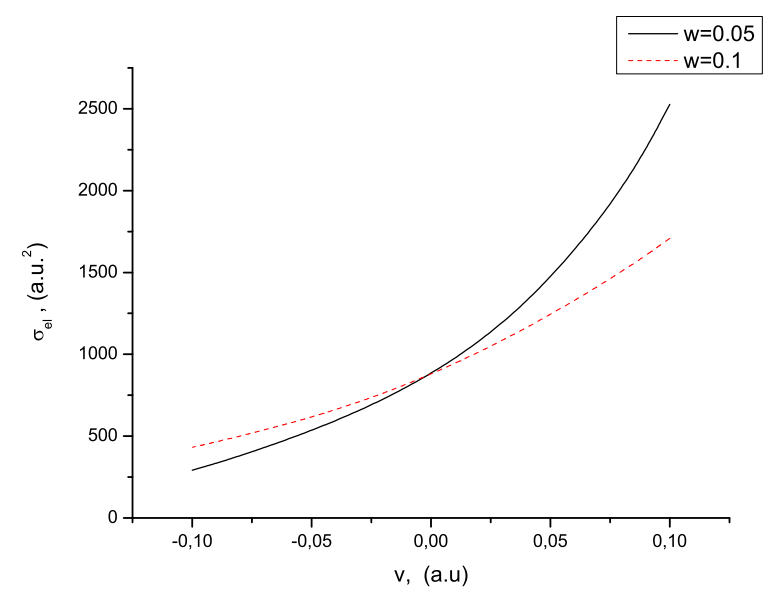

FIG. 5: Elastic cross-section as a function of the real part $v$ of the optical potential (24), calculated at the energy $\mathrm{E}=10^{-6}$ a.u.

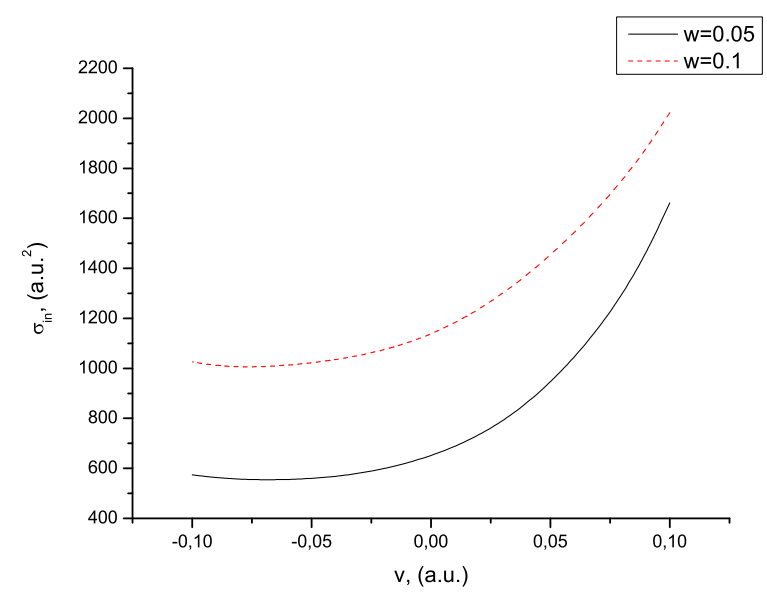

FIG. 6: Inelastic cross-section as function of the real part $v$ of the optical potential (24), calculated at the energy $\mathrm{E}=10^{-6}$ a.u.

ration of the short-range complex potential in the model calculations results in modification of $\delta_{R}$ and $\delta_{I}$. Strictly speaking, the effect of strong forces is not restricted to "direct" annihilation and scattering on the nuclear potential in the $H-\bar{H}$ channel. It appears also through modification of the final states and energies of Protonium. Neglecting for the moment these "second order" effects, one can use the well-known expression [31, 32] for the nuclear phase-shifts in the presence of Coulomb interaction to extract directly the phase-shift 
of interest:

$$
k\left[C(\eta) \cot \tilde{\delta}_{s c}+2 \eta h(\eta)\right]=-1 / \tilde{a}_{s c}
$$

where $\tilde{a}_{s c}$ is the Coulomb corrected nuclear scattering length, $\eta=-M / k$ ( $k$ is the c.m. proton-antiproton momentum) and functions $C(\eta), h(\eta)$ are given by

$$
\begin{gathered}
C(\eta)=\frac{2 \pi \eta}{\exp (2 \pi \eta)-1}, \\
h(\eta)=1 / 2\left[\Psi(-i \eta)+\Psi(i \eta)-\ln \left(\eta^{2}\right)\right] .
\end{gathered}
$$

In the above, $\Psi(x)=\Gamma^{\prime}(x) / \Gamma(x)$, where $\Gamma(x)$ is a standard gamma-function. Neglecting the leptonic energy in comparison with the value of Coulomb $p \bar{p}$ potential at the distance around $1 \mathrm{fm}$, we can put $k \rightarrow 0$ and get:

$$
\tilde{\delta}_{s c} \approx-2 \pi M \tilde{a}_{s c} .
$$

The values of the strong-force scatering lenghth $\tilde{a}_{s c}$ in the Kohno-Weise model [33, 34] are:

$$
\begin{aligned}
& \tilde{a}_{s c}(S=0)=(1.07-i 1.45) \cdot 10^{-5} \text { a.u., } \\
& \tilde{a}_{s c}(S=1)=(1.68-i 1.06) \cdot 10^{-5} \text { a.u. }
\end{aligned}
$$

while the corresponding nuclear phase-shifts are

$$
\begin{aligned}
& \tilde{\delta}_{s c}(S=0)=-0.06+i 0.08, \\
& \tilde{\delta}_{s c}(S=1)=-0.1+i 0.06
\end{aligned}
$$

where $S=0,1$ are the values of total nuclear spin.

The models I-IV include no information about strong forces. However we will show that, once the value of scattering length without any account of strong forces is known, one can also obtain the corrected value of this scattering length that includes the presence of strong forces. In fact, adding the nuclear phase-shifts (32) and (33) to the already discussed phase-shifts deduced from the rearrangement calculation of models I-IV

$$
\delta \rightarrow \delta+\tilde{\delta}_{s c},
$$

one gets the values of the scattering lengths and cross-sections, corresponding to simultaneous account of rearrangement and strong force effects. The above mentioned addition of phase-shifts can be done due to the fact that contributions to the phase from each of the 


\begin{tabular}{|c|c|c|c|c|}
\hline & Model I & Model II & Model III & Model IV \\
\hline$a$, a.u. & $5.2-\mathrm{i} 1.8$ & $8.2-\mathrm{i} 2.8$ & 5.6 -i3.7 & $7.7-\mathrm{i} 0$ \\
\hline$a^{s c}(S=0)$, a.u. & 5.6 -i2.2 & $8.3-\mathrm{i} 3.4$ & 5.8 -i4.0 & 8.4 -i0.4 \\
\hline$a^{s c}(S=1)$, a.u. & $5.8-\mathrm{i} 2.1$ & $8.6-\mathrm{i} 3.4$ & $6.0-\mathrm{i} 3.9$ & $8.1-\mathrm{i} 0.6$ \\
\hline$\delta$ & $0.696+\mathrm{i} 0.305$ & $0.136+\mathrm{i} 0.41$ & $0.638+\mathrm{i} 0.715$ & $0.287+\mathrm{i} 0$ \\
\hline$\sigma_{e l}$, a.u. $^{2}$ & 380 & 943 & 566 & 745 \\
\hline$\sigma_{e l}^{s c}(S=0)$, a.u. ${ }^{2}$ & 448 & 1018 & 623 & 889 \\
\hline$\sigma_{e l}^{s c}(S=1)$, a.u. ${ }^{2}$ & 472 & 1077 & 640 & 829 \\
\hline$\sqrt{E} \sigma_{i n}$, a.u. & 0.53 & 0.8 & 1.08 & 0 \\
\hline$\sqrt{E} \sigma_{i n}^{s c}(S=0)$, a.u. & 0.65 & 1.0 & 1.17 & 0.13 \\
\hline$\sqrt{E} \sigma_{i n}^{s c}(S=1)$, a.u. & 0.62 & 1.0 & 1.15 & 0.18 \\
\hline
\end{tabular}

TABLE I: Values of the short-range scattering lengths, phase-shifts and cross-sections (in a.u. ${ }^{2}$ ) calculated at the energy $E=10^{-6}$ a.u. with the parameters deduced from different models I-IV for $H-\bar{H}$ interaction. The superscript $s c$ corresponds to the account of the strong force in the presence of Coulomb field. The results were obtained by numerical solution of the one channel Schrödinger equation subject to the boundary condition given by eq. 1 with the phase $\delta$ from row 5 incremented by the strong force contribution given by eqs. (32, 33).

effects (strong force and rearrangement) come from very different distances. This is true until the effect of strong force on the final Pn states is not taken into account.

The results of our calculations for the elastic and inelastic scattering cross-sections with and without strong force, based on the parameters deduced from models I-IV are collected in Table I. We observe that the simultaneous account of rearrangement and strong-force effects significantly differs from the results where only one of these effects is taken into account.

The modification of cross-sections due to the strong force is significant when compared to the ratio of nuclear and atomic scales, which is on the order of $10^{-5}$. One can also see that this modification is model dependent. This fact is clear from our previous considerations of the cross-sections as function of the the short-range phase-shift. In particular, the change of the elastic cross-section due to the strong force is around $24 \%$ in model I, while it is $13 \%$ in model III. These models differ by the inelasticity parameter $\delta_{I}$ which is 2.3 times larger in model III as compared to model I. Consequently model III is less sensitive to any 
variation of phase, in particular the phase induced by strong interaction. The physical reason for the lack of sensitivity to the details of the short-range interaction with the increase of inelasticity parameter is clear. Indeed, the amplitude of the wave reflected back into the $H-\bar{H}$ channel that is generated at such small distances and "carries back" information about the nuclear forces is, in the case of strong absorption, exponentially small. Hence in the case of strong absorption the scattering length only weakly depends on the details of the short range interaction.

Let us mention here that the effect of nuclear forces on $H-\bar{H}$ interaction would be strongly enhanced in the vicinity of certain value of the real part of the short-range phase. According to (14) the largest variation of the scattering length with small variation of the phase $\delta_{R}$ is achieved when the following condition is fulfilled

$$
\pi / 8+\Omega+\delta_{R}=\pi v
$$

where $v$ is an integer number. As shown later in sec. III 4 this condition corresponds to the appearance of the loosely bound $H \bar{H}$ state. In particular, for the inelasticity parameter of model I $\left(\delta_{I}=0.3\right)$ the modification of the elastic cross-section due to the strong force in the near-resonance situation (eq. 34) would be from $\sigma_{e l}=3800 \mathrm{a.u.}^{2}$ without strong force to $\sigma_{e l}^{s c}(S=1)=2015$ a.u. ${ }^{2}$ and $\sigma_{e l}^{s c}(S=0)=2249$ with strong force, i.e. around $50 \%$.

\section{Isotope effect}

The oscillatory behavior of the cross sections gives rise to the strong isotope effect. Simple changing of the reduced mass alters the phases $\delta$ and $\Omega$ in eq. (14). The mass dependence of the phases can be easily established in the WKB approximation (25), from which it follows that the semiclassical phase is scaled as the square root of the reduced mass $M$. The change of the reduced mass from $M_{p} / 2$ to $2 / 3 M_{p}$ (where $M_{p}$ is the proton mass) corresponds to the replacement of hydrogen by deuterium and results in multiplication of the semiclassical phase by the factor $\sqrt{4 / 3}$

$$
\begin{gathered}
\Omega_{d}=\sqrt{4 / 3} \Omega \\
\delta_{d}=\sqrt{4 / 3}(\delta-\pi / 4)+\pi / 4 .
\end{gathered}
$$


Here the subscript $d$ refers to deuterium. In the above formula we took into account the Coulomb quantum correction $-\pi / 4$.

The numerical calculation of the scattering length while neglecting rearrangement and strong force (i.e. using potential $V_{l o c}(r)$ as the $D-\bar{H}$ interaction for $r<R_{s}$ ) gives

$$
a^{D \bar{H}}=-41.07 \text { a.u. }
$$

to be compared with the corresponding scattering length (without rearrangement and strong force) in the $H-\bar{H}$ case

$$
a^{H \bar{H}}=7.69 \text { a.u. }
$$

The account of rearrangement effects dramatically changes the situation.

The model calculations by Voronin and Carbonell [5] show that the scattering length changes from $a=5.2 .1-i 1.8$ a.u. for $\bar{H}-H$ to $15.0-i 11.6$ a.u. for the $\bar{H}-D$ case. In view of the demonstrated high sensitivity of the results to the short range interaction, very accurate calculations are required to obtain the reliable values of the cross-sections. However the prediction of a strong isotope effect has a general character and is a consequence of "weak absorption" in the $H-\bar{H}$ interaction. Note that in the opposite case of "strong absorption" the expression (19) predicts only weak monotonous dependence of the scattering length on the reduced mass via $a_{0} \sim \sqrt[4]{2 M C_{6}}$.

\section{The near-threshold quasimolecular states of $H-\bar{H}$}

The oscillating behavior of the scattering cross-section as a function of the phase $\delta$ has a clear physical meaning. The long range character of the attractive atom-antiatom interaction combined with the weak absorption supports the existence of long-lived highly excited $H-\bar{H}$ states [3, 8, 20, 26]. They manifest themselves as near-threshold singularities of the $S$-matrix and thus strongly affect the low energy scattering. By changing the phase through varying the strength of interaction, changing the reduced mass of the system, including additional decay channels, etc., the position of such states can be tuned to be at the threshold, inducing a rapid increase of the cross-sections as a function of the tuning parameter. The $S$-matrix of the finite range potential can be expressed through the position of all its poles via the following expression [35]:

$$
S=\prod_{k} \frac{z_{k}+k}{z_{k}-k} \exp (-2 i k \bar{a})
$$


where $z_{k}$ is the pole of $S$-matrix and $\bar{a}$ is independent on $k$.

If there is a $S$-matrix pole $z$, situated much closer to the threshold than other poles, we can approximate the infinite product by one term only:

$$
S(k) \approx \frac{z+k}{z-k} \exp (-2 i k \bar{a})
$$

In the limit of small $k$ the expression (36) becomes $S(k)=1+2 k / z-2 i k \bar{a}$, from where one gets the connection between the scattering length and $z$

$$
a=i / z+\bar{a} .
$$

When there are two (or more) poles at similar distance from the threshold (bound and virtual states) one should take into account the contribution of the number of equidistant poles of the $S$-matrix so that the expression for the scattering lengths becomes $a=i \sum_{k} 1 / z_{k}+\bar{a}$.

It was shown in [12, 36] that the above expression is applicable in case of scattering on potentials with $1 / r^{6}$ tail. In such a case the constant $\bar{a}$ in the one-pole expansion is $\bar{a}=a_{0}$, with $a_{0}$ given by eq. (8) . Therefore the position of the pole in the one-pole expansion of the $S$-matrix is given by

$$
z=i /\left(a-a_{0}\right)
$$

This relation is valid in the case when the distance between poles is much bigger then $z$. Substituting the expression for the scattering length given by eq. (17) we get

$$
z=\frac{i}{a_{0}} \tan (\delta+\Omega+\pi / 8) .
$$

Taking into account that the near-threshold states are possible only in case of weak absorption $\left(\delta_{I} \ll 1\right)$ we obtain

$$
z=\frac{i}{a_{0}} \tan \left(\delta_{R}+\Omega+\pi / 8\right)-\frac{\delta_{I}}{a_{0} \cos ^{2}\left(\delta_{R}+\Omega+\pi / 8\right)} .
$$

The above equation is valid only for the expansion with respect to the single nearestthreshold pole, which gives the dominant contribution to the scattering length.

From the above equation it is clear that due to the inelasticity of scattering characterized by $\delta_{I}$, the near-threshold $S$-matrix poles are shifted to the left from the imaginary axis of the complex $k$-plane. These shifted poles in the 2 -nd quadrant of the complex $k$-plane can be interpreted as localized (bound) states with an inelastic width, while the poles in the 3-rd quadrant are the virtual states or resonances with inelastic width. (The distinction between 
resonances and virtual states can be obtained by a continuous decrease of inelasticity. In the limit of zero inelasticity the virtual states come to the negative imaginary axis of the $k$-plane, while the resonances are manifested as pairs of poles in the 3-rd and 4-th quadrants.)

The condition for the appearance of the new state now means that the pole comes from the third to the second quadrant of the complex $k$-plane. At the moment when the pole of the $S$-matrix crosses the real (negative) axis of the complex k-plane one has $\operatorname{Im} z=0$. According to eq. (39) this is equivalent to condition (34):

$$
\delta_{R}+\Omega+\pi / 8=\pi v
$$

where $v$ is an integer number. Numerical integration of the adiabatic potential $V_{a d}$ gives the WKB phase $\Omega=19.383$ so that the numerical value of $\delta_{R}$ (by modulus $\pi$ ) corresponding to the resonance is $\delta_{R}=-0.926$.

We have shown that the previously introduced "resonance" condition (34) is indeed the condition for the appearance of a new state at the threshold. At the moment of appearance of the new state the scattering length is:

$$
a_{\text {res }}=a_{0}\left(1-i \frac{\cosh \left(\delta_{I}\right)}{\sinh \left(\delta_{I}\right)}\right)
$$

and its imaginary part attains its maximum value. In case of weak absorption $\left(\delta_{I} \ll 1\right)$ it can be written as

$$
\operatorname{Im} a_{r e s}=-\frac{a_{0}}{\delta_{I}} .
$$

At the same time, the variation $\Delta a$ due to a small change of the real part of the phase-shift $\Delta \delta_{R}$ attains its maximum value:

$$
\Delta a=\frac{2 a_{0} \Delta \delta_{R}}{\cosh \left(\delta_{I}\right)}
$$

Hence the effect of additional interactions (e.g. the account of additional decay channels, strong force, etc.) in the near-resonant situation will result in a fast change of the real part of the scattering length. The point at which the $S$-matrix pole crosses the real axis of the complex $k$-plane can be obtained by comparing formulas (39) and (41):

$$
z_{\text {res }}=-\frac{\delta_{I}}{a_{0}}
$$

It is determined entirely by the inelasticity parameter. The larger is the inelasticity parameter, the larger is the shift of the $S$-matrix pole to the left from the origin of the complex $k$ -plane, and the smaller is its influence on the scattering cross-sections. 
The energy of the near-threshold state is:

$$
E_{b}=\frac{z^{2}}{2 M}=-\frac{\tan ^{2}\left(\delta_{R}+\Omega+\pi / 8\right)}{2 M a_{0}^{2}}-\frac{i \delta_{I} \tan \left(\delta_{R}+\Omega+\pi / 8\right)}{M a_{0}^{2} \cos ^{2}\left(\delta_{R}+\Omega+\pi / 8\right)} .
$$

If the pole $z$ is very close to the real axis of the complex $k$-plane, so that $\delta_{R}+\Omega+\pi / 8=\pi v+\varphi$ and $\varphi \ll \delta_{I}$, the expression (42) should be modified:

$$
E_{0}=\frac{\left(-\delta_{I}+i \varphi\right)^{2}}{2 M a_{0}^{2}}
$$

Here $\varphi$ measures the de-tuning of the phase from the exact resonance. The state at the threshold is extremely extended spatially and has a very small width:

$$
\Gamma=\frac{2 \varphi \delta_{I}}{2 M a_{0}^{2}}
$$

The detailed study of the near-threshold states in $1 / R^{6}$ potential can be found in [17, 36]. In the above cited papers the higher order expansion in $a_{0} /\left(a-a_{0}\right)$ for the energy of nearthreshold state is given:

$$
E_{0}=-\frac{1}{2 M\left(a-a_{0}\right)^{2}}\left(1+\frac{0.918 a_{0}}{a-a_{0}}-\frac{0.947 a_{0}^{2}}{\left(a-a_{0}\right)^{2}}\right)
$$

Taking into account (7) we get:

$$
E_{0}=-\frac{\tan ^{2}(\delta+\Omega+\pi / 8)}{2 M a_{0}^{2}}\left(1+0.918 \tan (\delta+\Omega+\pi / 8)-0.947 \tan ^{2}(\delta+\Omega+\pi / 8)\right)
$$

We observe that once the scattering length $a$ is fixed by a certain model, the spectrum of near-threshold quasi-molecular states is also fixed (up to the total number of $H-\bar{H}$ states with given hadron angular momentum). Indeed, the value of the scattering length uniquely determines the short-range complex phase-shift $\delta$ and thus the boundary condition (1), while the solution of the Schrödinger equation with given adiabatic potential and fixed boundary condition (1) gives the spectrum (up to the number of states). The validity of this statement is restricted only by the approximation that our boundary condition (11) is energy independent, i.e. it is valid for energies $E \ll \varepsilon_{m} \approx 0.05$ a.u.. We calculate the positions of the bound states nearest to the threshold, as they are fixed by the scattering lengths of various models. These values are collected in Table II . We also present the position of the same states when the spin dependent strong force is taken into account. One can see that the shift of the states due to the account of nuclear forces is model dependent and is of order $10^{-5}$ a.u. for the first loosely bound state. The splitting $\Delta E^{s c}$ of the triplet and singlet spin 


\begin{tabular}{|c|c|c|c|c|}
\hline & Model I & Model II & Model III & Model IV \\
\hline$E, 10^{-5}$ a.u. & $-10.1-i 5.6$ & $-2.2-i 3.2$ & $-3.8-i 13.1$ & $-4.9-i 0$ \\
\hline$E^{s c}(S=0) 10^{-5}$ a.u. & $-8.4-i 6.7$ & $-1.4-i 3.3$ & $-0.7-i 12.8$ & $-4.3-i 0.8$ \\
\hline$E^{s c}(S=1) 10^{-5}$ a.u. & $-7.8-i 6.1$ & $-1.3-i 2.9$ & $-0.8-i 11.5$ & $-3.9-i 0.6$ \\
\hline
\end{tabular}

TABLE II: Energy of the state nearest to the threshold. The first row collects the values without account of the strong force, the second and the third row collects the values with account of the strong force in spin states $S=0$ and $S=1$ correspondingly. The results were obtained by numerical solution of the one channel Schrödinger equation subject to the boundary condition given by eq. 11 with the phase including the strong force contribution, together with the square-integrability requirement.

states is on the order of $10^{-6}$ a.u., which is one order less than the inelastic width due to rearrangement.

It is useful to see, how the position of the pole that is nearest to the threshold changes with the short-range phase-shift $\delta$. Such an evolution of the poles in models I-III is presented on Fig:7. Each of the shown trajectories corresponds to a certain fixed value of inelasticity parameter $\delta_{I}$. The evolution of the $S$-matrix pole along the trajectory can be understood as a gradual "switching on" the real part of effective interaction, responsible for rearrangement (while its imaginary part is kept fixed). At certain value of the phase $\delta_{R}$ the pole crosses the real axis of the k-plane, and the new vibrational state (quasi-bound) appears in the spectrum of $H-\bar{H}$ system. With increasing the real part of effective interaction (and correspondingly the phase $\delta_{R}$ ) this pole goes far away from the threshold, becoming deeper bound state. At the same time a new pole with the negative imaginary momentum (virtual state) is approaching the real axis. This is reflected in oscillatory behavior of the elastic and inelastic cross-sections.

We conclude by emphasizing that the resonant dependence of the cross-sections on the interaction parameters (such as real and imaginary short-range phase-shift) is of purely quantum mechanical origin and occurs only in the case of weak absorption. This resonance behavior reflects the existence of the spectrum of narrow, near-threshold, quasi-molecular states of $H-\bar{H}$. 


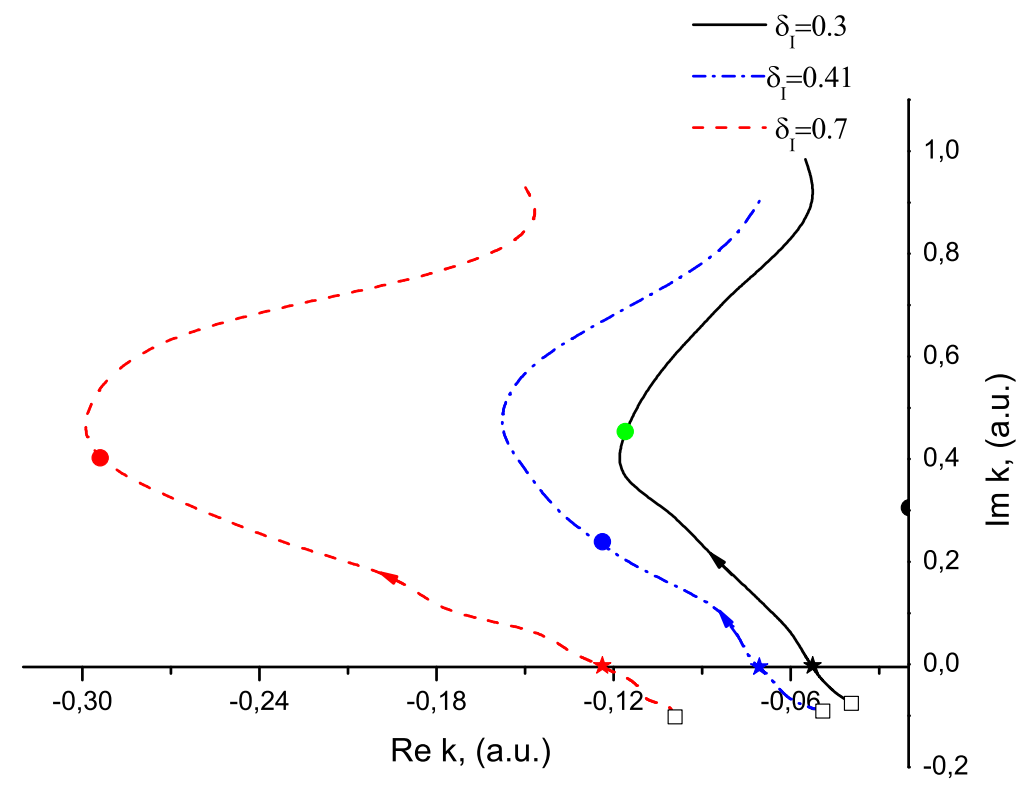

FIG. 7: Evolution of the poles of the $S$-matrix in the complex $k$-plane as function of the real part of the short-range phase-shift, $-\pi / 2 \leq \delta_{R} \leq \pi / 2$. Solid line corresponds to the fixed inelasticity parameter $\delta_{I}=0.3$ (model I), dash-dotted line - inelasticity parameter $\delta_{I}=0.41$ (model II) and dashd line - inelasticity parameter $\delta_{I}=0.7$ (model III). An empty square marks the virtual state, a star marks the point where the $S$-matrix pole crosses the real axis of the complex k-plane, a circle - the position of the bound state corresponding to the "true" value of the phase-shift $\delta_{R}$, deduced for each model from the corresponding scattering length. In calculations, the diverging character of the virtual states was tempered using the technique of exterior complex scaling [37]. The arrow shows the direction of the pole evolution with increasing $\delta_{R}$.

\section{CONCLUSIONS}

We have presented an overview of the $H-\bar{H}$ collisions based on the analytical treatment of the asymptotic potential tail in conjunction with the parametrization of the contribution from the inner part of the interaction. We show analytically that for a realistic value of the inelasticity parameter $\delta_{I}$ (the imaginary part of the short-range scattering phase induced by the rearrangement and the strong-force interaction) a rich spectrum of narrow near-threshold $H \bar{H}$ states exists. These states, corresponding to the near-threshold singularities of the $S$ matrix, determine the behavior of the elastic and inelastic cross-sections. The latter exhibit 
high sensitivity to even small perturbation of the $H-\bar{H}$ interaction or its approximate treatment. We predict important physical effects such as a strong isotope effect (i.e. the resonance-like dependence of elastic and inelastic cross-sections on the reduced mass of the system) and demonstrate a significant dependence of both elastic and inelastic $H-\bar{H}$ crosssections on the nuclear potential.

\section{ACKNOWLEDGMENT}

The research was performed under support from the Wenner-Gren Foundations, the Royal

Swedish Academy of Sciences, the Swedish Research Council and the Russian Foundation for Basic Research grant 02-02-16809. 
[1] W. Kolos, D.L. Morgan, D.M. Schrader, and L. Wolniewicz. Phys. Rev. A, 11:1792, 1975.

[2] E.L. Surkov G.V. Shlyapnikov, J.T.M. Walraven. Hyperfine Interactions, 76:31, 1993.

[3] A. Voronin and J. Carbonell. Hyperfine Interactions, 115:143, 1998.

[4] P. Froelich. Quantum Chemistry of Antimatter. Adv. Qunt. Chem., 41:185, 2002 and the references therein.

[5] A. Voronin and J. Carbonell. Nucl. Instr. and Methods B, 214:139, 2004.

[6] P. Froelich, S. Jonsell, A. Saenz, B. Zygelman, and A. Dalgarno. Phys. Rev. Lett., 84:4577, 2000 .

[7] S. Jonsell, A. Saenz, P. Froelich, A. Dalgarno, and B. Zygelman. Phys. Rev. A, 64:052712, 2001.

[8] B. Zygelman, A. Saenz, P. Froelich, and S. Jonsell. Phys. Rev. A, 69:042715, 2004.

[9] Y. Liu G.D.R. Martin E.A.G. Armour, C.W. Chamberlain. Nucl. Instr. Meth. B, 221:1, 2004.

[10] Krzysztof Strasburger. J.Phys. B: At. Mol. Opt. Phys., 37:4483, 2004.

[11] Christopher Eltschka Michael J. Moritz and Harald Friedrich. Phys. Rev. A, 64:022101, 2001.

[12] V.V. Flambaum G.F.Gribakin. Phys. Rev. A, 48:546, 1993.

[13] A. Dalgarno R. Côté, E. J. Heller. Phys. Rev. A, 53:234, 1996.

[14] K. Strasburger. J. Phys. B, 35, 2002.

[15] M.F. Mott and H.S.W. Massey. The theory of atomic collisions. Oxford, Clarendon Press, 1965.

[16] C. Harabati V.V. Flambaum, G.F.Gribakin. Phys. Rev. A, 59:1998, 1999.

[17] Bo Gao. Phys. Rev. A, 58:4222, 1998.

[18] J. Trost R. Cóte, H. Friedrich. Phys. Rev. A, 56:1781, 1997.

[19] B. Zygelman A.Yu. Voronin, P. Froelich. Phys. Rev. A, 72:062903, 2005.

[20] P. Froelich, B. Zygelman, A. Saenz, S. Jonsell, S. Eriksson, and A. Dalgarno. Few-Body Systems, 34:63, 2004.

[21] S. Jonsell, A. Saenz, P. Froelich, B. Zygelman, and A. Dalgarno. J. Phys. B, 37:1195, 2004.

[22] E.A.G. Armour and C.W. Chamberlain. J. Phys. B, 35, 2002.

[23] Y Liu E A G Armour and A Vigier. J.Phys. B: At. Mol. Opt. Phys., 38, 2005.

[24] P.A. Sinha and A.S. Ghosh. Europhys. Lett., 49:558, 2000. 
[25] P.K. Sinha and A.S. Ghosh. Phys. Rev. A, 68:022504, 2003.

[26] A. Voronin and J. Carbonell. Phys. Rev. A, 57:4335, 1998.

[27] A. Voronin and J. Carbonell. Nucl. Phys. A, 689:529, 2001.

[28] A. Prozorov G. Plunien L. Labzowsky, V. Sharipov and G. Soff. Phys. Rev. A, 72:022513, 2005 .

[29] S. Jonsell, A. Saenz, and P. Froelich. Low Energy Hydrogen - Antihydrogen Collisions. Nuclear Physics A, 663:959c, 2000.

[30] S. Jonsell, A. Saenz, P. Froelich, B. Zygelman, and A. Dalgarno. Including the Strong Nuclear Force in Antihydrogen Scattering Calculations. Can. J. Phys. , 83:435, 2004.

[31] Watson K.M. Goldberger, M.I. Collision theory. New York: Wiley, 1964.

[32] T.L. Trueman. Nucl. Phys., 26:57, 1961.

[33] Weise W. Kohno, M. Nucl. Phys. A, 454:429, 1986.

[34] J.-M. Richard J.Carbonell and S. Wycech. Z. Phys. A -Hadrons and Nuclei, 343:325, 1998.

[35] R. G. Newton. Scattering Theory of Waves and Particles. Springer-Verlag, New York, 1982.

[36] Bo Gao. J.Phys. B: At. Mol. Opt. Phys., 37:4273, 2004.

[37] J.D. Morgan and B. Simon. J. Phys. B, 14:L167, 1981. 